\title{
Quality of Experience Assessment of Video Streaming
}

\author{
Moomal Memon ${ }^{1}$, Asif Ali Laghari ${ }^{2 *}$, Zaffar Ahmed Shaikh $^{3}$, S.Basil Xavier ${ }^{4}$ \\ ${ }^{1}$ Department of IT, Quaid-e-Awam University of Engineering, Science \& Technology, Nawabshah, Pakistan, \\ moomalmemon10@gmail.com \\ ${ }^{2}$ Department of Computer Science, Sindh Madressatul Islam University, Karachi, Pakistan, \\ asif.laghari@smiu.edu.pk \\ ${ }^{3}$ Benazir Bhutto Shaheed University, Karachi, Pakistan, zashaikh@bbsul.edu.pk \\ ${ }^{4}$ Department of Computer Science and Engineering, Karunya Institute of Technology and Science, Coimbatore, \\ India, basilxavier.s@gmail.com
}

\begin{abstract}
This study aims to determine the user's satisfaction level of online streaming by using different web browsers. At the client layer, the assessment of the user's QoE is conducted by evaluating the performance of three web browsers (Google Chrome, Mozilla Firefox, and Internet Explorer). We took the subjective test by conducting different experiments with the users and ask the users to assign ratings on the provided questionnaires, and from those ratings, we calculated results in the form of Mean Opinion Score.
\end{abstract}

Key words: Quality of experience (QoE); video streaming; web browser.

\section{INTRODUCTION}

Over the internet or network, the perception of video streaming has been growing for many years [1]. This video streaming has been predominantly significant because of its acceptance by many users around the world. Nowadays video streaming has become a vast topic in the research area because everybody wants from the providers to deliver rich multimedia content over the internet or network. The areas for research under the topic video streaming include packet loss, delay, packet reordering, bandwidth allocation of different video streaming qualities include $1080 \mathrm{p}$, 720p, 360p, 240p, etc. The performance of the web browsers as well in terms of video streaming matters a lot.

The increasing demand for video streaming and watching videos without downloading $(\mathrm{VoD})$, which leads to low quality and delay in video streaming shakes the QoE. Poor quality and delay in streaming cannot be controlled by service providers [2]. Usually, Users face problems regarding the quality of the video, which has been affected either by network traffic, video compressed by social networks or by the poor performance of web browsers in terms of page loading time, speed, buffering [3].

In recent years $\mathrm{QoE}$ has also become a vast research theme in telecommunication; it is an analysis of human experience when interacting with technology and business point of view. The QoE improves performance such as user's effectiveness, efficiency, and satisfaction during usage of particular products or services [4]. The demand for multimedia applications is promptly increasing every day. Nowadays people are quality meters and their needs and perception carry big issues for service providers because it is hard to measure user satisfaction during the usage of particular products or services. The purpose of this study is to analyze the impact of web browser to improve the QoE of the users.

\section{LITERATURE REVIEW}

Liang Zhou has investigated a problem that QoE based cloud services are under considerable attention because of most of the current work primarily emphasis on reducing the waiting time. But how to make an effectual delay is the actual deal. For this purpose, the researcher studied the components of the user's reaction and enterprise a delay announcement scheme based on users QoE by taking an objective user response function [5].

Amerini et al. have identified the source of the videos uploaded on social networks like Facebook, Twitter by using five-finger extraction methods and a novel 
method to form a compound fingerprint to attain meaningful results. The result shows that it is still conceivable to identify the source of the device [6]. Lorentzen et al. studied the experience of the user regarding web pages in terms of security for authentication purposes. After conducting several experiments, it has been analyzed that user's tolerance level is quite higher when security feature is involved [7].

Laghari et al. proposed some QoE frameworks for multimedia services. These frameworks have been studied in terms of monitoring, data analysis, reporting, and policy change support and deployment parameters. It has been analyzed that not all of the frameworks support both objective and subjective QoE assessment except EQoM, which supports all most all the significant components of multimedia services [8].

Balachandran et al. emphasize the importance of the relationship between the web QoE and network characteristics to help the network operators to realize when and where web QoE is degraded by the network conditions. Researchers developed a model that helps network operators to observe web QoE using standard radio network metrics alone and improve the network features for better QoE [9]. Hoßfeld et al. proposed the challenge of evaluating and demonstrating QoE for online video streaming grounded on TCP streaming. The result shows that the $\mathrm{QoE}$ valuation technique that is extremely operative not only for online video for other applications also is crowdsourcing [10].

\section{EXPERIMENT FOR QOE ASSESSMENT OF WEB BROWSERS}

$\mathrm{HD}$ videos take additional time to load into a web page as compared to low-quality videos because the big size of the file requires more bandwidth to transmit the data from server to user. It lowers the interest of a user when it takes a lot of time in the loading of a web page. At the client layer, we selected three most commonly used web browsers i.e., Google Chrome, Mozilla Firefox and Internet Explorer. The users were guided to access the videos, which were posted on Facebook, Vimeo and Google+ at both scales i.e. $360 \mathrm{p}$, and $720 \mathrm{p}$ by using all three web browsers. This experiment was conducted to observe users QoE towards the performance of web browsers in terms of page loading time, video buffering and browser speed.

The LCD of 21 inches with $1280 \times 800$ resolutions is used according to suggestions by ITU-R, for displaying videos. Experiments were conducted at different times on different days, among them, $44 \%$ were female students and $56 \%$ were male students, aged between 20 to 30 years. The majority of them were students of IT and BSCS department and some of them were postgraduate students from MS (SE), MS (IT), and MS (CS). The students were asked to watch the videos by using web browsers and provide feedback in the form of ratings on the given questionnaires.

\subsection{MEAN OPINION SCORE (MOS)}

Mean Opinion Score is a standard offered by ITU as given in Table 1 [2]. It is the average value of all the ratings that we got from users. We used MOS for conducting subjective evaluation from users. Questionnaires were provided to the users ranges from 1 to 5 (1 for Bad quality, 2 for Poor, 3 for Fair, 4 for Good and 5 for Excellent quality).

Table 1: Mean Opinion Score

\begin{tabular}{|c|c|c|}
\hline Quality & MOS & Perception \\
\hline Bad & 1 & Very Annoying \\
\hline Poor & 2 & Annoying \\
\hline Fair & 3 & Slightly Annoying \\
\hline Good & 4 & Perceptible \\
\hline Excellent & 5 & Imperceptible \\
\hline
\end{tabular}

If the loading time, buffering of video and speed of browsers satisfy the user and they think that the browsers are better, the user assigns rating as excellent, and if the user is merely satisfied with the quality, he/she will assign a rating to the video as fair. If they think and observe, the overall performance is worse they will assign a bad rating to the particular browser. 


\section{RESULTS AND DISCUSSION}

\subsection{PERFORMANCE OF WEB BROWSERS AT} 360P

Table 2 shows the MOS of the Google Chrome browser at $360 \mathrm{p}$ and participants assigned 4.5 on Facebook, 4.2 on Vimeo and 4.8 on Google+ using Google Chrome for 360p videos.

Table 2. MOS of Google Chrome 360p

\begin{tabular}{|c|c|}
\hline Social Networks & MOS \\
\hline Facebook & 4.5 \\
\hline Vimeo & 4.2 \\
\hline Google+ & 4.8 \\
\hline
\end{tabular}

It has been shown in this table that for Google Chrome at 360p, we got better results on all three social networks and participants were satisfied with the performance of Google Chrome at 360p. Figure 1 shows the MOS of Google Chrome at 360p.

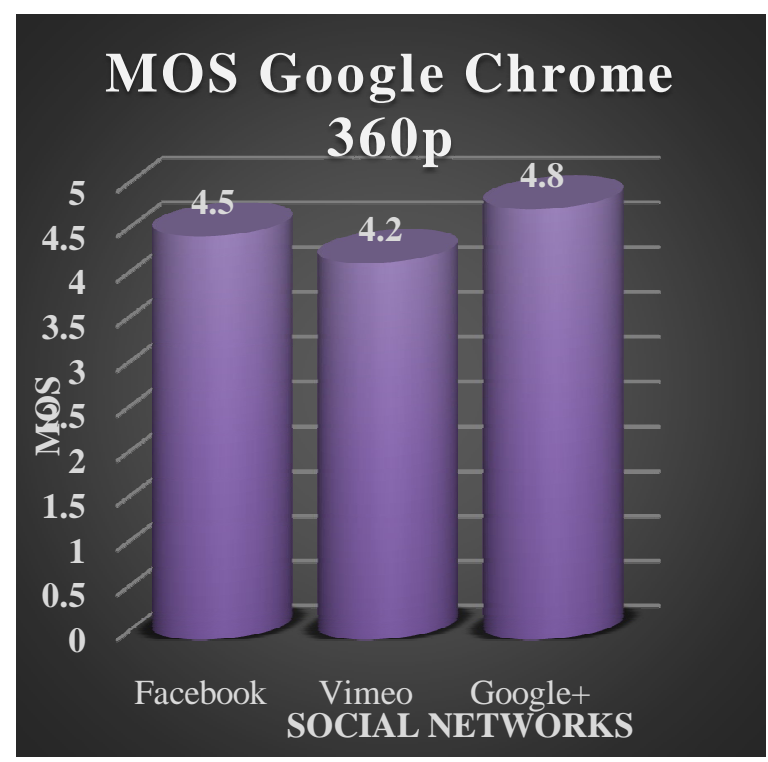

Figure 1. MOS Google Chrome 360p

Table 3 Shows the MOS of Mozilla Firefox at 360p. The participants assigned 4.8 on Facebook, 4.0 on Vimeo, and 4.7 on Google+ using Mozilla Firefox for $360 \mathrm{p}$ videos.
Table. 3 MOS of Mozilla Firefox 360p

\begin{tabular}{|c|c|}
\hline Social Networks & MOS \\
\hline Facebook & 4.8 \\
\hline Vimeo & 4.0 \\
\hline & \\
\hline Google+ & \\
& 4.7 \\
\hline
\end{tabular}

MOS from the above table shows that like Google Chrome, participants assigned good ratings to Mozilla Firefox as well. Hence Mozilla Firefox provides satisfactory QoE to the users at 360p. Figure 2 shows MOS graph of Mozilla Firefox for 360p Videos.

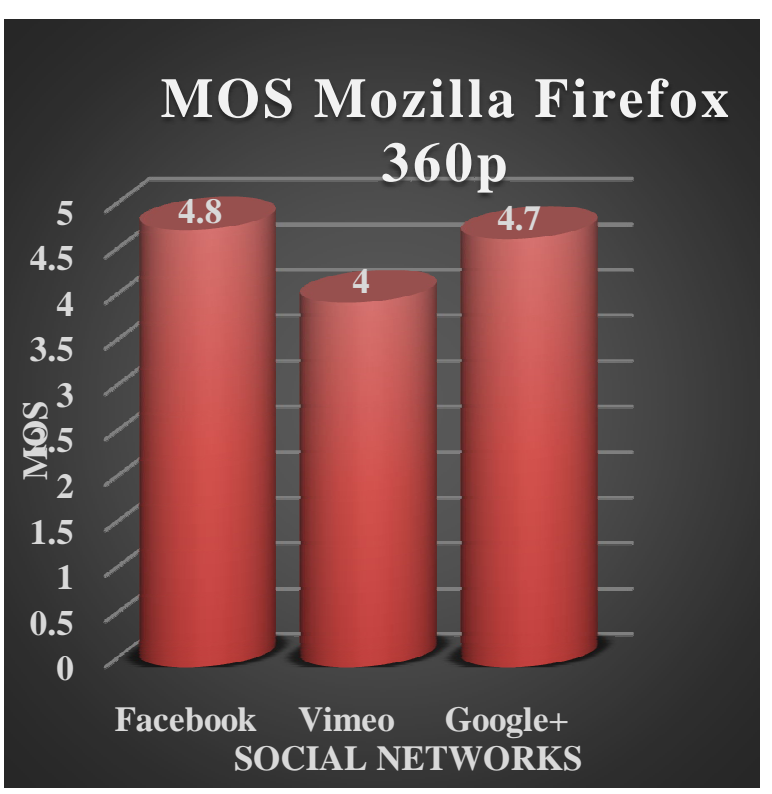

Figure 2 MOS Mozilla Firefox 360p 
Table 4 shows the MOS of Internet Explorer at 360p. Participants assigned 4.3 on Facebook, 3.8 on Vimeo and 4.4 on Google+ using Mozilla Firefox for 360p videos. Figure 3 shows the MOS graph of Internet Explorer for 360 videos on Facebook, Vimeo and Google+

Table 4 MOS of Internet Explorer 360p

\begin{tabular}{|c|c|}
\hline Social Networks & MOS \\
\hline Facebook & 4.3 \\
\hline Vimeo & 3.8 \\
\hline & \\
\hline Google+ & 4.4 \\
\hline
\end{tabular}

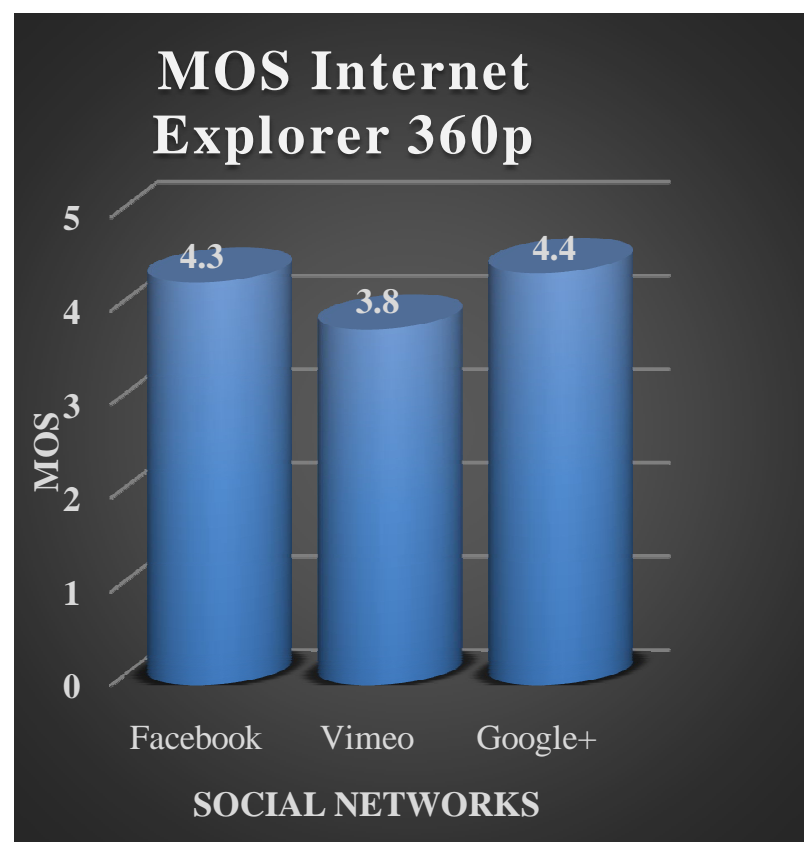

Figure 3 MOS Internet Explorer 360p

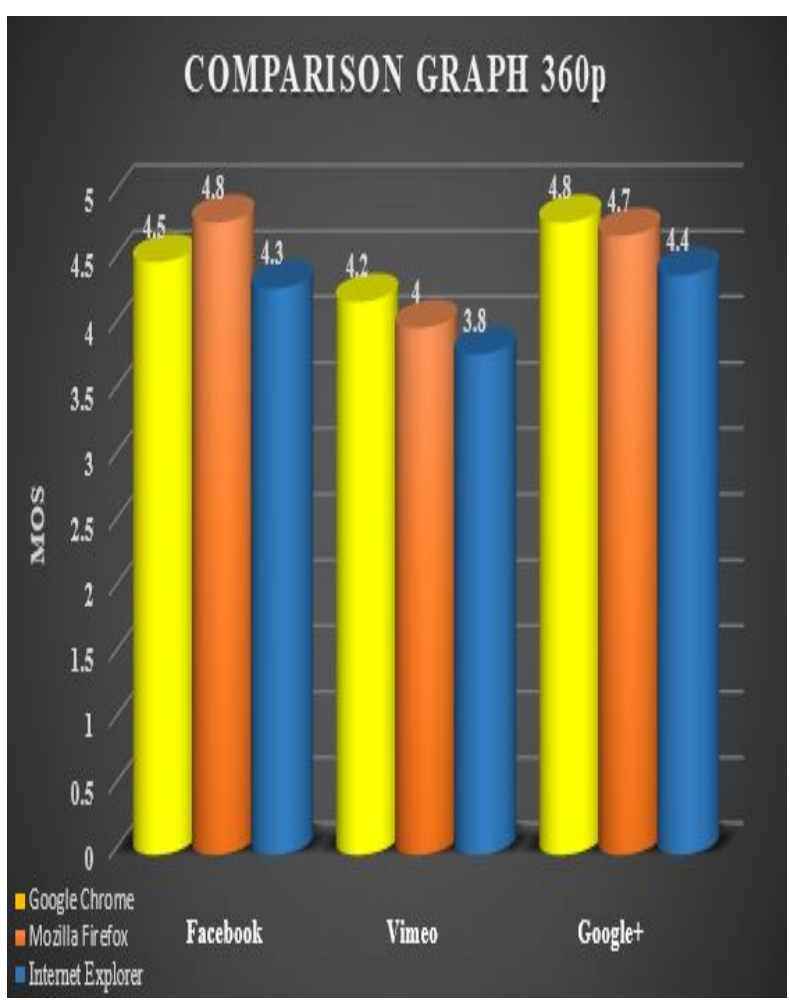

Figure 4 Comparison Graph

Figure 4 shows the comparison graph of the MOS of Google Chrome, Mozilla Firefox and Internet Explorer at 360p.t

The resulting graph shows that users satisfied with the performance of Google Chrome and Mozilla Firefox as compared to Internet Explorer. The user assigned better ratings for 360p videos on Facebook, Vimeo and Google+ because videos at 360p have low quality and as we know that low-quality videos take less time to load into a webpage that eventually speed-up the performance of web browsers.

\subsection{PERFORMANCE OF WEB BROWSERS AT} 720P

Table 5 shows the MOS of the Google Chrome browser at 720p. Participants assigned 3.3 on Facebook, 3.0 on Vimeo and 4.0 on Google+ using Google Chrome for 720p videos. Figure 5 shows the MOS graph of Google Chrome at 720p. 
Table 5 MOS of Google Chrome 720p

\begin{tabular}{|c|c|}
\hline Social Networks & MOS \\
\hline Facebook & 3.5 \\
\hline Vimeo & 2.9 \\
\hline & \\
\hline Google+ & 3.8 \\
\hline
\end{tabular}

Table 6 MOS of Mozilla Firefox 720p

\begin{tabular}{|c|c|}
\hline Social Networks & MOS \\
\hline Facebook & 3.3 \\
\hline Vimeo & 3.0 \\
\hline & \\
\hline Google+ & 4.0 \\
\hline
\end{tabular}

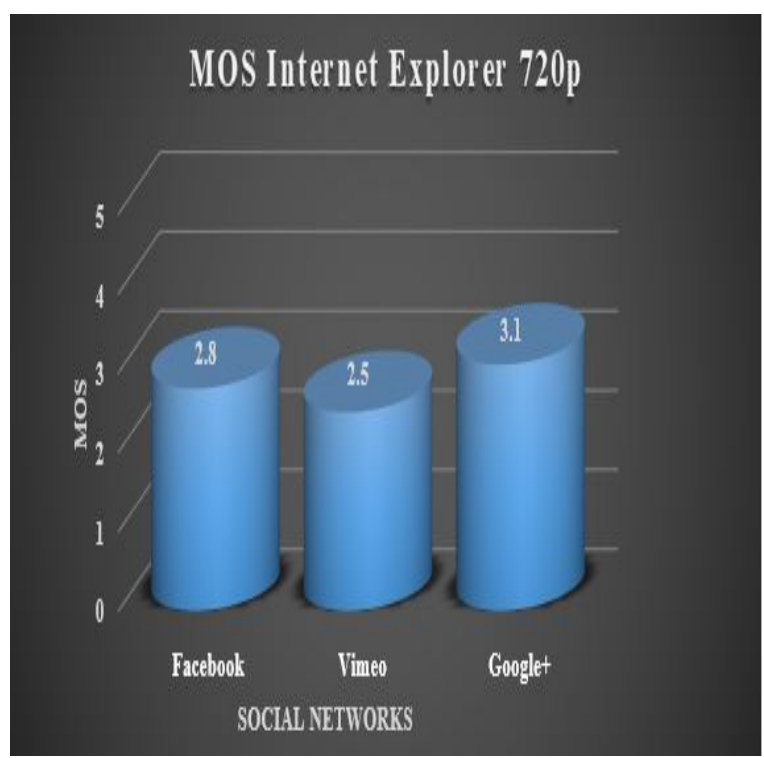

Figure. 5 MOS of Google Chrome 720p

Table 6 shows the MOS of the Mozilla Firefox browser at 720p. Participants assigned 3.5 on Facebook, 2.9 on Vimeo and 3.8 on Google+ using Google Chrome for 720 p videos. Figure 6 shows the MOS graph of Mozilla Firefox at 720p.

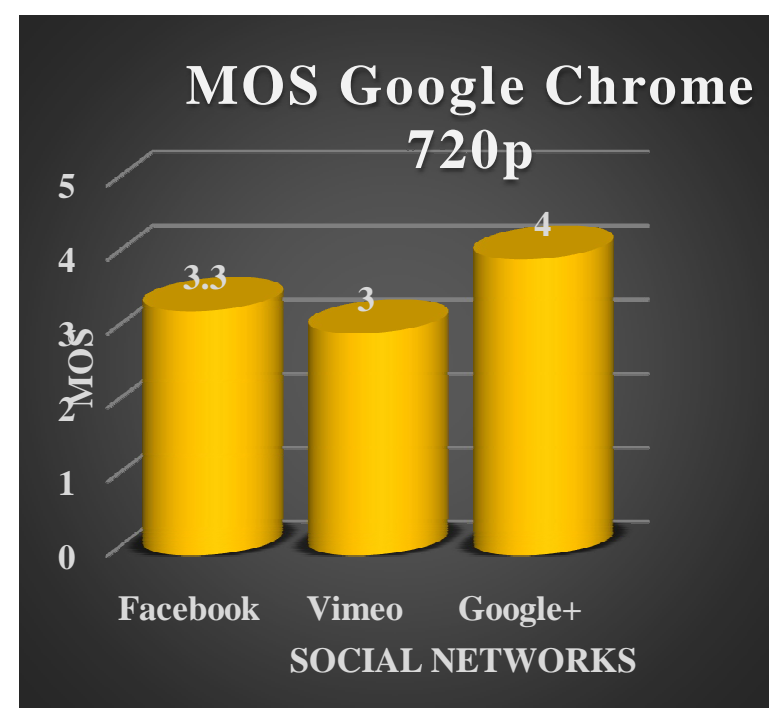

Figure 6 MOS of Mozilla Firefox 720p 
Table 7 shows the MOS of the Internet Explorer browser at $720 \mathrm{p}$. Participants assigned 2.8 on Facebook, 2.5 on Vimeo and 3.1 on Google+ using Google Chrome for 720p videos. Figure 7 shows the MOS graph of Internet Explorer at 720p.

Table. 7 MOS of Internet Explorer 720p

\begin{tabular}{|c|c|}
\hline Social Networks & MOS \\
\hline Facebook & 2.8 \\
\hline Vimeo & 2.5 \\
\hline Google+ & \\
\hline & 3.1 \\
\hline
\end{tabular}

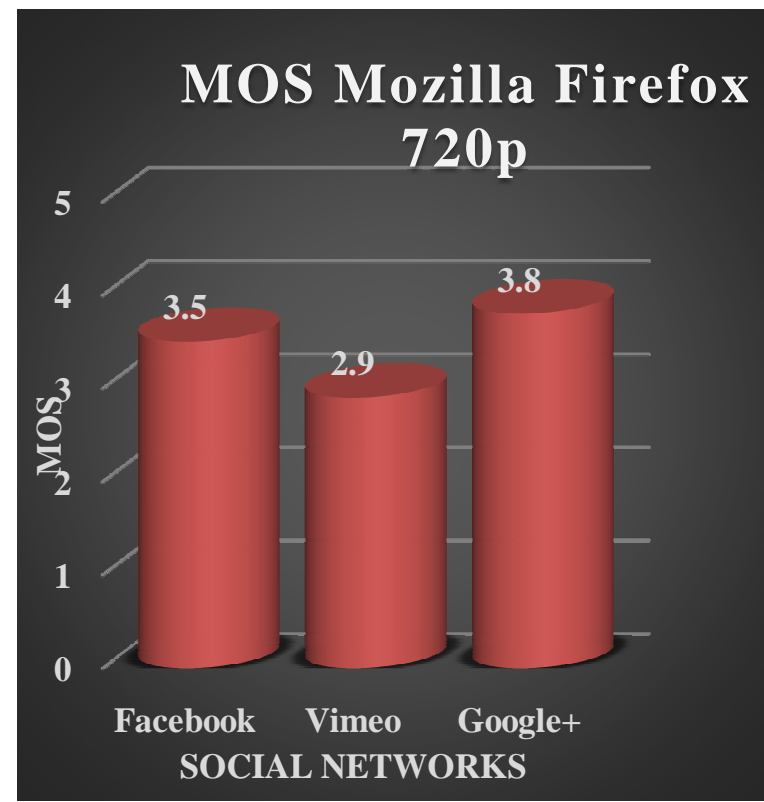

Figure. 7 MOS Internet Explorer 720p

Figure 8 shows the comparison graph of Google Chrome, Mozilla Firefox and Internet Explorer for 720p videos on Facebook, Vimeo and Google+

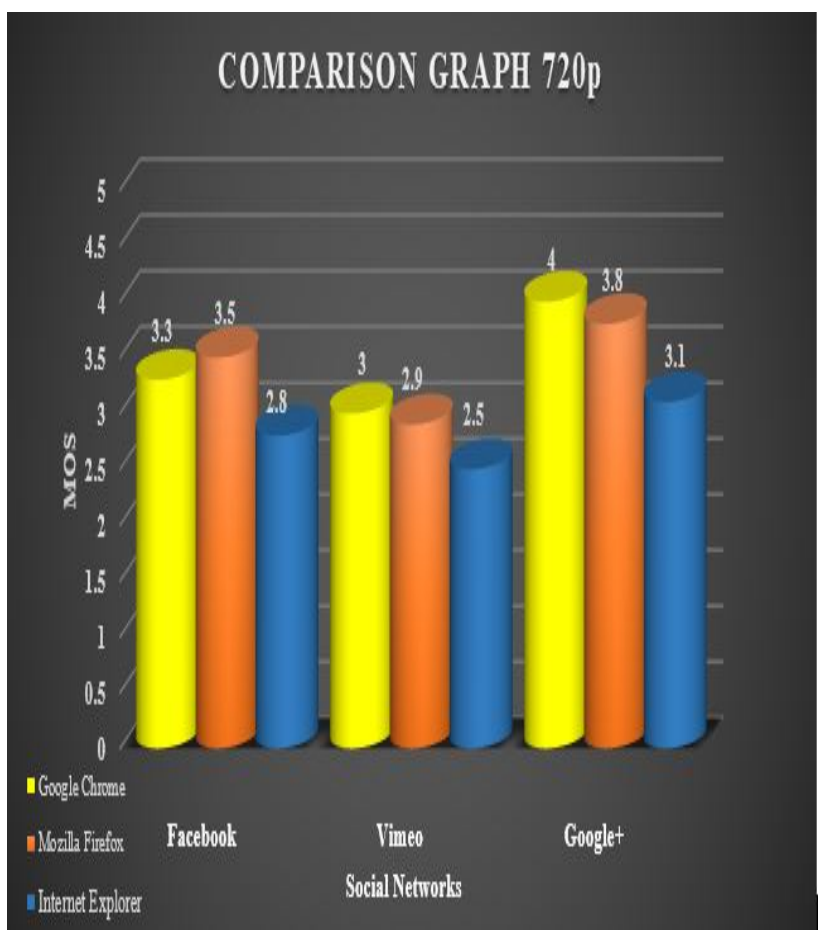

Figure. 8 Comparison Graph

It has been shown in the results that we have not got many satisfactory ratings on $720 p$ videos for browsers, because high-quality videos take more time to load into a webpage, and it requires more bandwidth to transfer the data from server to the client.

\section{CONCLUSION}

In this paper, the performance of browsers was evaluated at the client layer and we got satisfactory ratings for Google Chrome and Mozilla Firefox as well. On the $360 \mathrm{p}$ scale, we got satisfactory ratings on all the three browsers as compare to the $720 \mathrm{p}$ scale, because videos with the resolution of 720p scale have high quality and videos with high quality take more time to load.

\section{CONFLICT OF INTEREST}

Authors did not have any conflict of interest.

\section{REFERENCES}

[1] Broom, Simon, Xiao-Si Wang, and James Schofield. "Method for assessing the perceived quality of adaptive video streaming." U.S. Patent 10,541,894, issued January 21, 2020. 
[2] Laghari, Asif Ali, Hui He, Asiya Khan, and Sajida Karim. "Impact of video file format on quality of experience (QoE) of multimedia content." 3D Research 9, no. 3 (2018): 39.

[3] Laghari, Asif Ali, Hui He, Muhammad Shafiq, and Asiya Khan. "Application of quality of experience in networked services: Review, trend \& perspectives." Systemic Practice and Action Research 32, no. 5 (2019): 501-519.

[4] Karn, Nabin Kumar, Hongli Zhang, Feng Jiang, Rahul Yadav, and Asif Ali Laghari. "Measuring bandwidth and buffer occupancy to improve the QoE of HTTP adaptive streaming." Signal, Image and Video Processing 13, no. 7 (2019): 1367-1375.

[5] Wang, Ying, Peilong Li, Lei Jiao, Zhou Su, Nan Cheng, Xuemin Sherman Shen, and Ping Zhang. "A data-driven architecture for personalized QoE management in $5 \mathrm{G}$ wireless networks." IEEE Wireless Communications 24, no. 1 (2016): 102-110.

[6] Laghari, Asif Ali, Hui He, and Muhammad Ibrahim Channa. "Measuring effect of packet reordering on quality of experience (QoE) in video streaming." 3D Research 9, no. 3 (2018): 30.

[7] Amerini, Irene, Roberto Caldelli, Andrea Del Mastio, Andrea Di Fuccia, Cristiano Molinari, and Anna Paola Rizzo. "Dealing with video source identification in social networks." signal processing: Image communication 57 (2017): 1-7.

[8] Laghari, Asif Ali, Hui He, ShehnilaZardari, and Muhammad Shafiq. "Systematic analysis of quality of experience (QoE) frameworks for multimedia services." IJCSNS 17, no. 5 (2017): 121.

[9] Crespi, Noel, B. Molina, and C. E. Palau. "QoE aware service delivery in distributed environment." In 2011 IEEE Workshops of International Conference on Advanced Information Networking and Applications, pp. 837-842. IEEE, 2011.

[10] Hoßfeld, Tobias, Michael Seufert, Matthias Hirth, Thomas Zinner, Phuoc Tran-Gia, and Raimund Schatz. "Quantification of YouTube QoE via crowdsourcing." In 2011 IEEE International Symposium on Multimedia, pp. 494-499. IEEE, 2011. 